For Internal Distribution Only

Accelerator Division

Alternating Gradient Synchrotron Department

BROOKHAVEN NATIONAL LABORATORY

Upton, New York 11973

Accelerator Division

Technical Note

AGS/AD/Tech. Note No. 421

THE J-10 DUMP BUMP

E. Bleser

October 24, 1995 


\section{THE J-10 DUMP BUMP}

\section{SUMMARY}

This note documents the design of the $3 / 2$ lambda bump that will be installed to bump the AGS beam into the new dump at J-10.

\section{THE J-10 BUMP}

The new bump will be very similar to the existing $3 / 2$ lambda bumps. It will use backleg windings on the main AGS magnets. Table 1 specifies its construction. Column 1 lists the magnets involved, column 2 specifies the magnet type, column 3 indicates whether the bump dipole field adds or subtracts from the main dipole field, column 4 gives the hook-up polarity in the standard AGS nomenclature, column 5 gives the number of turns on the backleg ( 6 on a short magnet, 5 on a long magnet), column 6 gives the magnet specification for running the standard version of the AGS MAD modeling program, and column 7 gives the phase advance from the exit of magnet I-10 to the exits of the other magnets. The cable on the magnets is $4 / 0$ flex with a resistance of about 5.4 milliohms per magnet. At 400 amperes DC this cable will reach a temperature of about 100 degrees C.

\section{THE MODELING RESULTS}

Figure 1 shows the closed orbit in the AGS when the $15 \mathrm{GeV} \mathrm{MAD} \mathrm{model}{ }^{1}$ is run with a dipole field error of $1 \%$ in the magnets of Table 1. Figure 2 is a detail of Figure 1. Table 2 tabulates for reference the data plotted in Figure 2. The markers show the beam positions at the PUE's (The actual PUE readings are at present in error by $+4 \mathrm{~mm}$ ). Elaborate modeling studies have been done and the details may be reported elsewhere.

\section{OPERATIONS}

Figure 3 shows the current ${ }^{2}$ needed in the bump to produce a displacement of $20 \mathrm{~mm}$ at the exit of the main magnet $\mathrm{J}-10$. The bump performance can be summarized by the equation:

$$
\mathbf{I}=\mathbf{X} * \mathbf{P} *[.2653+0.001 * \mathbf{P}]
$$

where: $\mathrm{I}=$ bump current in Amperes:

$\mathrm{X}=$ Inward displacement of the closed orbit at the exit of Main Magnet J-10 in millimeters;

$\mathrm{P}=$ momentum in $\mathrm{GeV} / \mathrm{c}$.

Displacements at other points in the orbit can be scaled from Table 2.

${ }^{1}$ Standard models of our machine are maintained by E. Auerbach on the Apollo System in directory "users/Models". Current versions of the MAD program are maintained by J. Niederer.

${ }^{2}$ Field vs current data for the AGS main magnets is available from $\mathrm{R}$. Thern. 
TABLE 1. THE J-10 BUMP

\begin{tabular}{|c|c|c|c|c|c|c|}
\hline MAGNET & TYPE & $\begin{array}{c}\text { CHANGE IN } \\
\text { BEND }\end{array}$ & $\begin{array}{c}\text { HOOKUP } \\
\text { POLARITY }\end{array}$ & $\begin{array}{c}\text { BACKLEG } \\
\text { TURNS }\end{array}$ & $\begin{array}{c}\text { MAD } \\
\text { SEQUENCE }\end{array}$ & $\begin{array}{c}\text { dMUX } \\
2^{*} \text { PI }\end{array}$ \\
\hline $\mathrm{I}-10$ & BF & - & B & 6 & BF[36] & 0.000 \\
\hline $\mathrm{l}-11$ & BD & - & B & 6 & BD[33] & 0.072 \\
\hline $\mathrm{J}-4$ & CD & + & A & 5 & CD[38] & 0.534 \\
\hline $\mathrm{J}-5$ & AF & + & A & 5 & AF[19] & 0.620 \\
\hline $\mathrm{J}-18$ & CF & + & A & 5 & CF[40] & 1.027 \\
\hline $\mathrm{J}-19$ & BD & + & A & 6 & BD[39] & 1.059 \\
\hline $\mathrm{K}-12$ & BD & - & B & 6 & BD[42] & 1.551 \\
\hline $\mathrm{K}-13$ & CF & - & B & 5 & CF[41] & 1.575 \\
\hline
\end{tabular}

$Q x=8.720$

TABLE 2. THE J_10 BUMP CLOSED ORBIT

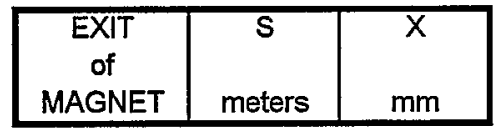

\begin{tabular}{|l|r|r|}
\hline 110 & 570.155 & -0.9 \\
\hline 111 & 575.209 & 1.5 \\
\hline 112 & 577.825 & 3.8 \\
\hline PUE I12 & 578.112 & 4.1 \\
\hline 113 & 580.822 & 6.2 \\
\hline 114 & 584.734 & 6.9 \\
\hline PUE I14 & 585.021 & 6.8 \\
\hline 115 & 587.731 & 6.6 \\
\hline I16 & 591.642 & 9.1 \\
\hline 117 & 594.639 & 11.4 \\
\hline I18 & 598.551 & 9.9 \\
\hline PUE I18 & 598.838 & 9.6 \\
\hline I19 & 601.167 & 7.5 \\
\hline 120 & 603.783 & 7.0 \\
\hline
\end{tabular}

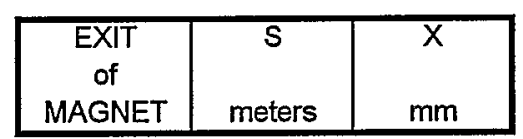

\begin{tabular}{|c|c|c|c|c|c|}
\hline $\mathrm{J1}$ & 608.837 & 7.5 & $\overline{\mathrm{K} 1}$ & 676.093 & 7.3 \\
\hline$\sqrt{2}$ & 611.453 & 5.4 & $\mathrm{~K} 2$ & 678.71 & 7.2 \\
\hline PUE J2 & 611.74 & 5.1 & PUE K2 & 678.997 & 7.1 \\
\hline J3 & \begin{tabular}{|l|}
614.45 \\
\end{tabular} & 2.6 & $\mathrm{~K} 3$ & 681.707 & 6.7 \\
\hline$\sqrt{14}$ & 618.362 & -0.5 & $\mathrm{~K} 4$ & 685.618 & 9.0 \\
\hline PUE J4 & 618.649 & -0.8 & PUE K4 & 685.905 & 9.3 \\
\hline J5 & 621.359 & -3.4 & $\mathrm{~K} 5$ & 688.615 & 11.2 \\
\hline$\sqrt{16}$ & 625.27 & -6.4 & K6 & 692.527 & 9.6 \\
\hline J7 & 628.267 & -8.3 & $\overline{\mathrm{K} 7}$ & 695.524 & 6.9 \\
\hline \begin{tabular}{|l|}
$J 8$ \\
\end{tabular} & 632.179 & -14.3 & $\mathrm{~K} 8$ & 699.435 & 6.4 \\
\hline PUE J8 & 632.466 & -15.0 & PUE K8 & 699.722 & 6.5 \\
\hline$\overline{\mathrm{J9}}$ & 634.795 & -19.1 & $\mathrm{Kg}$ & 702.051 & 6.6 \\
\hline J10 & 637.411 & -19.2 & K10 & 704.667 & 5.2 \\
\hline \begin{tabular}{|l} 
J10 SS MP \\
\end{tabular} & \begin{tabular}{l|l}
638.935 \\
\end{tabular} & -17.4 & K11 & 709.722 & 1.0 \\
\hline
\end{tabular}




\section{J10 BUMP, CLOSED ORBIT}

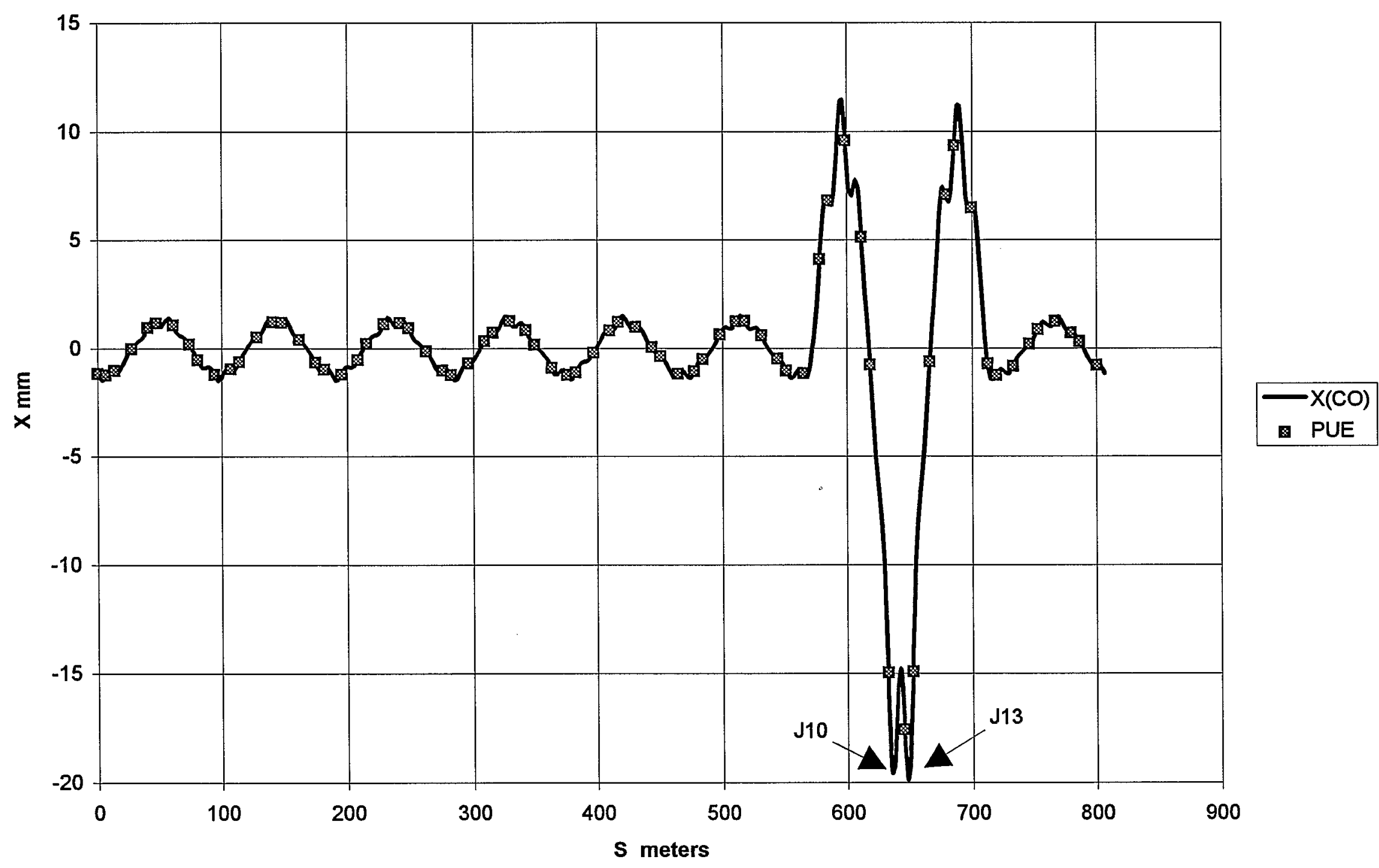




\section{J10 BUMP, CLOSED ORBIT}

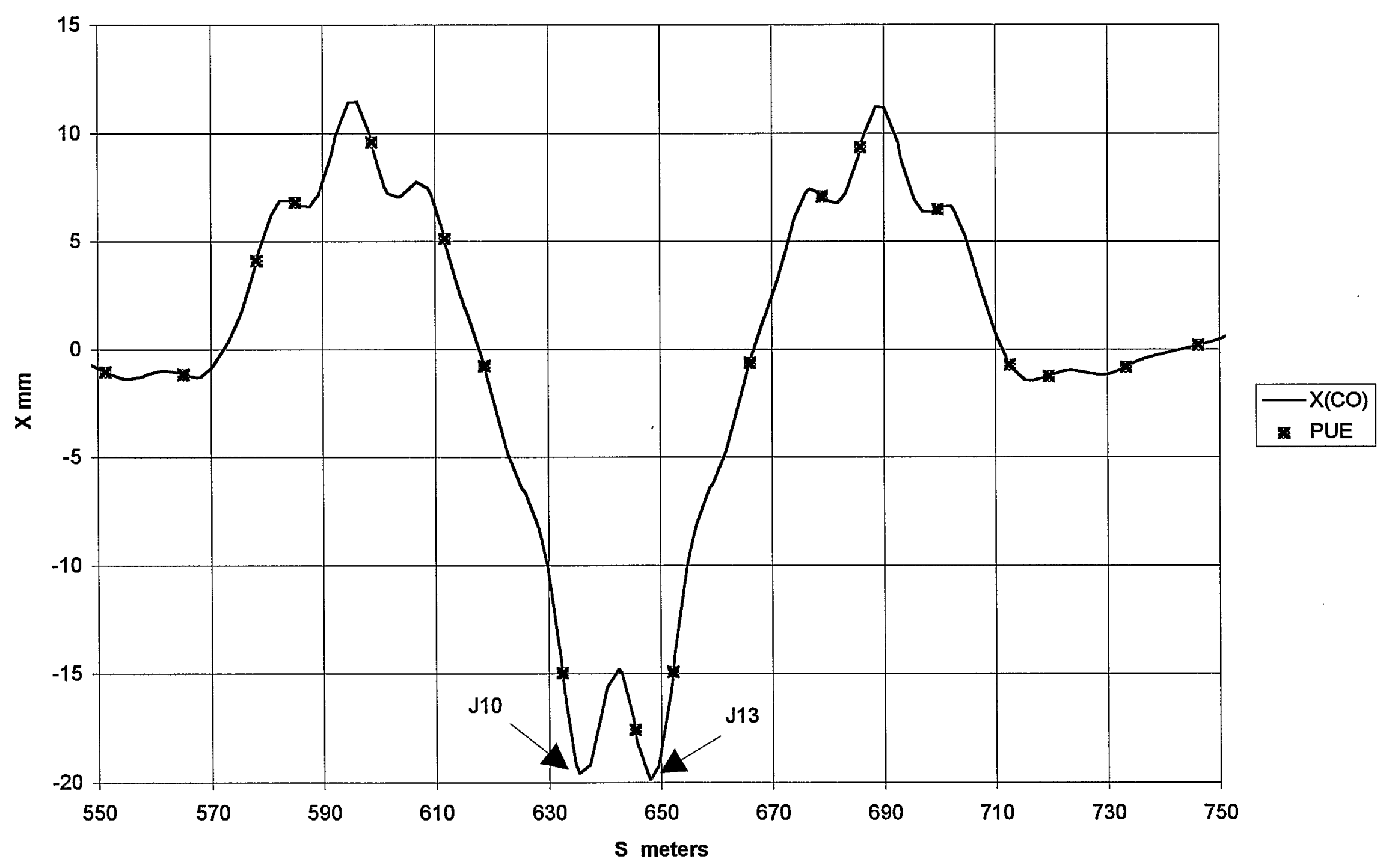




\section{J-10 BUMP CURRENT for 20 mm BUMP}

1

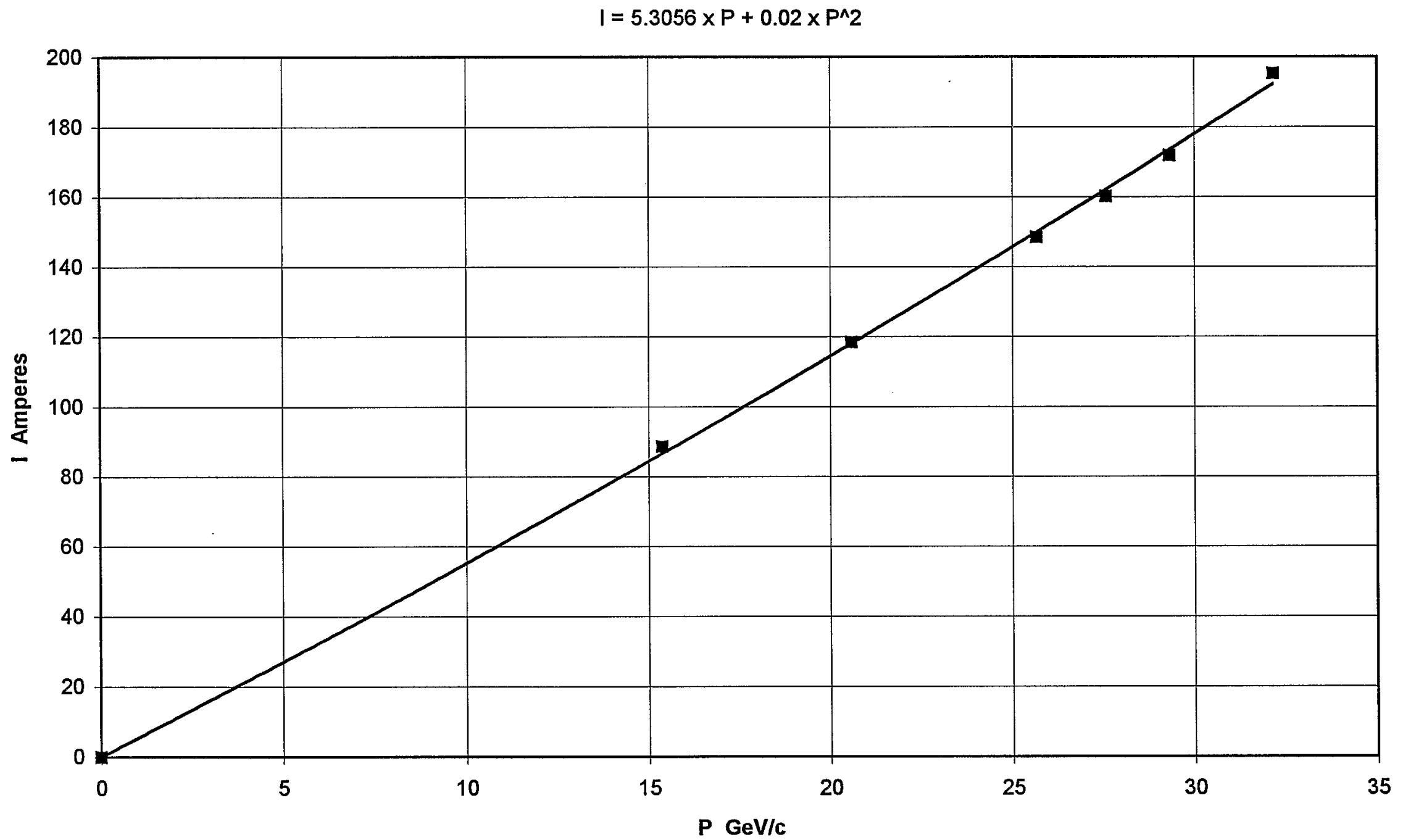

